\title{
Timing of eating across ten European countries - results from the European Prospective Investigation into Cancer and Nutrition (EPIC) calibration study
}

Ena Huseinovic ${ }^{1, *}$, Anna Winkvist ${ }^{1,2}$, Heinz Freisling ${ }^{3}$, Nadia Slimani $^{3}$, Heiner Boeing ${ }^{4}$, Genevieve Buckland $^{5}$, Lukas Schwingshackl ${ }^{4}$, Anja Olsen ${ }^{6}$, Anne Tjønneland ${ }^{6}$, Magdalena Stepien ${ }^{3}$, Marie-Christine Boutron-Ruault ${ }^{7,8}$, Francesca Mancini 7,8 , Fanny Artaud ${ }^{7,8}$, Tilman Kühn9 ${ }^{9}$ Verena Katzke ${ }^{9}$, Antonia Trichopoulou 10,11, Androniki Naska $^{10,11}$, Philippos Orfanos ${ }^{10,11}$, Rosario Tumino ${ }^{12}$, Giovanna Masala ${ }^{13}$, Vittorio Krogh ${ }^{14}$, Maria Santucci de Magistris ${ }^{15}$, Marga C Ocké ${ }^{16}$, Magritt Brustad ${ }^{17}$, Torill Enget Jensen 17, Guri Skeie ${ }^{17}$, Miguel Rodríguez-Barranco 18,19', José María Huerta '19, Eva Ardanaz 19,20,21, José Ramón Quirós ${ }^{22}$, Paula Jakszyn ${ }^{23}$, Emily Sonestedt ${ }^{24}$, Ulrika Ericson ${ }^{24}$, Maria Wennberg ${ }^{2}$, Timothy J Key ${ }^{25}$, Dagfinn Aune ${ }^{26}$, Elio Riboli ${ }^{26}$, Elisabete Weiderpass $27,28,29,30$ and Heléne Bertéus Forslund ${ }^{1}$

${ }^{1}$ Department of Internal Medicine and Clinical Nutrition, The Sahlgrenska Academy, University of Gothenburg, Box 459, SE-405 30, Gothenburg, Sweden: ${ }^{2}$ Department of Public Health and Clinical Medicine, Nutritional Research, Umeå University, Umeå, Sweden: ${ }^{3}$ Nutrition and Metabolism Section, International Agency for Research on Cancer, Lyon, France: ${ }^{4}$ Department of Epidemiology, German Institute of Human Nutrition, Nuthetal, Germany: ${ }^{5}$ Unit of Nutrition and Cancer, Cancer Epidemiology Research Programme, Catalan Institute of Oncology (ICO-IDIBELL), Barcelona, Spain: ${ }^{6}$ Danish Cancer Society Research Center, Copenhagen, Denmark: ${ }^{7}$ Université Paris-Saclay, Université Paris-Sud, UVSQ, CESP, INSERM, Villejuif, France: ${ }^{8}$ Institut Gustave Roussy, Villejuif, France: ${ }^{9}$ German Cancer Research Center (DKFZ), Division of Cancer Epidemiology, Heidelberg, Germany: ${ }^{10}$ Hellenic Health Foundation, Athens, Greece: " $\mathrm{WHO}$ Collaborating Center for Nutrition and Health, Unit of Nutritional Epidemiology and Nutrition in Public Health, Department of Hygiene, Epidemiology and Medical Statistics, School of Medicine, National and Kapodistrian University of Athens, Athens, Greece: ${ }^{12}$ Cancer Registry and Histopathology Department, 'Civic - M.P. Arezzo' Hospital, ASP, Ragusa, Italy: ${ }^{13}$ Cancer Risk Factors and Life-Style Epidemiology Unit, Cancer Research and Prevention Institute - ISPO, Florence, Italy: ${ }^{14}$ Epidemiology and Prevention Unit, Fondazione IRCCS Istituto Nazionale dei Tumori, Milan, Italy: ${ }^{15} \mathrm{AOU}$ Federico II, Naples, Italy: ${ }^{16} \mathrm{Centre}$ for Nutrition, Prevention and Health Services, National Institute for Public Health and the Environment (RIVM), Bilthoven, The Netherlands: ${ }^{17} \mathrm{UiT}$ The Arctic University of Norway, Tromsø, Norway: ${ }^{18}$ Escuela Andaluza de Salud Pública, Instituto de Investigación Biosanitaria ibs. GRANADA, Hospitales Universitarios de Granada/Universidad de Granada, Granada, Spain: ${ }^{19} \mathrm{CIBER}$ de Epidemiología y Salud Pública (CIBERESP), Madrid, Spain: ${ }^{20}$ Navarra Public Health Institute, Pamplona, Spain: ${ }^{21}$ IdiSNA, Navarra Institute for Health Research, Pamplona, Spain: ${ }^{22}$ Public Health Directorate, Asturias, Spain: ${ }^{23}$ Unit of Nutrition, Environment and Cancer, L'Hospitallet de Llobregat, Barcelona, Spain: ${ }^{24}$ Department of Clinical Sciences in Malmö, Lund University, Lund, Sweden: ${ }^{25}$ Cancer Epidemiology Unit, Nuffield Department of Population Health, University of Oxford, Oxford, UK: ${ }^{26}$ Department of Epidemiology and Biostatistics, School of Public Health, Imperial College London, London, UK: ${ }^{27}$ Department of Community Medicine, Faculty of Health Sciences, UIT The Arctic University of Norway, Tromsø, Norway:

${ }^{28}$ Department of Research, Cancer Registry of Norway, Institute of Population-Based Cancer Research, Oslo, Norway: ${ }^{29}$ Department of Medical Epidemiology and Biostatistics, Karolinska Institutet, Stockholm, Sweden:

${ }^{30}$ Genetic Epidemiology Group, Folkhälsan Research Center, and Faculty of Medicine, University of Helsinki, Helsinki, Finland 


\begin{abstract}
Objective: To examine timing of eating across ten European countries.

Design: Cross-sectional analysis of the European Prospective Investigation into Cancer and Nutrition (EPIC) calibration study using standardized $24 \mathrm{~h}$ diet recalls collected during 1995-2000. Eleven predefined food consumption occasions were assessed during the recall interview. We present time of consumption of meals and snacks as well as the later:earlier energy intake ratio, with earlier and later intakes defined as 06.00-14.00 and 15.00-24.00 hours, respectively. Type III tests were used to examine associations of sociodemographic, lifestyle and health variables with timing of energy intake.

Setting: Ten Western European countries.

Subjects: In total, 22985 women and 13035 men aged 35-74 years ( $n$ 36 020).

Results: A south-north gradient was observed for timing of eating, with later consumption of meals and snacks in Mediterranean countries compared with Central and Northern European countries. However, the energy load was reversed, with the later:earlier energy intake ratio ranging from 0.68 (France) to 1.39 (Norway) among women, and from 0.71 (Greece) to 1.35 (the Netherlands) among men. Among women, country, age, education, marital status, smoking, day of recall and season were all independently associated with timing of energy intake (all $P<0.05$ ). Among men, the corresponding variables were country, age, education, smoking, physical activity, BMI and day of recall (all $P<0 \cdot 05$ ).

Conclusions: We found pronounced differences in timing of eating across Europe, with later meal timetables but greater energy load earlier during the day in Mediterranean countries compared with Central and Northern European countries.
\end{abstract}

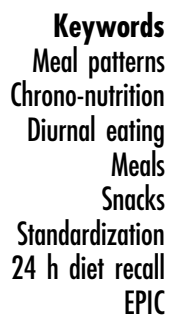

In Europe, CVD, diabetes and cancer are some of the leading causes of death and account for a large proportion of the disease burden in the region ${ }^{(1)}$. Still, these conditions are all largely preventable by tackling modifiable risk factors, including unhealthy diet ${ }^{(2,3)}$. More recently, the emerging field of 'chrono-nutrition' has been emphasized as research suggests that eating at the 'wrong time' may impair metabolism and that misalignment between timing of eating and endogenous circadian systems is associated with increased inflammation and adverse health outcomes $^{(4-8)}$. For example, observational studies have shown that later-night eaters have an increased risk of $\mathrm{CHD}^{(9)}$ and that shift workers have a higher risk of developing type 2 diabetes compared with day workers, which is believed to reflect greater consumption of energy intake during the night ${ }^{(10,11)}$. Furthermore, high evening relative to morning energy intake has been positively associated with $\mathrm{BMI}^{(12)}$ and individuals consuming a larger proportion of daily energy at dinner ( $\geq 48 \%$ of total energy intake) have been found to have an increased risk of obesity, metabolic syndrome and non-alcoholic fatty liver disease, even after adjusting for a set of covariates including breakfast skipping, total energy intake and dietary fibre ${ }^{(13)}$. Thus, not only the amount and content of food intake, but also elements such as timing of food intake need to be considered as potential risk factors for diet-related chronic diseases.

In the USA, the American Dietetic Association states that greater consumption of energy intake during the day may be preferable to evening consumption for weight management ${ }^{(14)}$. In the European guidelines for obesity management in adults, general advice is given to avoid breakfast skipping and eating at night ${ }^{(15)}$. However, despite the potential health implications of timing of eating, associated individual characteristics and their variation among different populations, cultures and geographical regions have rarely been investigated. This is likely due to the inconsistent approaches used to examine meal patterns, including a lack of standardized terminology, heterogeneity in how meal patterns are analysed and the wide range of assessment methods used, making interpretation and comparability between studies and countries problematic $^{(16,17)}$. Nevertheless, from a public health perspective, information on sociodemographic, sociocultural and lifestyle characteristics associated with timing of eating is needed to guide the development of evidencebased dietary recommendations and identify at-risk groups for preventive actions.

In a recent report, we utilized the standardized and homogeneous methodology used in the European Prospective Investigation into Cancer and Nutrition (EPIC) calibration study to characterize meal patterns across ten European countries. We found distinct differences in meals patterns across Europe, with marked diversity for intake frequency and proportional energy contribution from meals and snacks between Mediterranean and Central/Northern European countries ${ }^{(18)}$. However, differences in timing of eating were not covered in that report. 
Such analyses have the potential to further map geographical differences in meal patterns and provide a valuable resource and benchmark for Europe. Hence, the aim of the present paper was to describe meal timetables and to examine the timing of eating and its association with sociodemographic, lifestyle and health-related characteristics in women and men from ten European countries.

\section{Methods}

\section{Study population}

The present paper is based on data collected within the EPIC calibration study, a nested study within EPIC undertaken during 1995-2000. Details of the rationale, design and populations of EPIC, and of the calibration study, have been described elsewhere ${ }^{(19,20)}$. In short, EPIC is a multicentre cohort study aimed at investigating the association between dietary, biological, lifestyle and environmental factors in the aetiology of cancer and other chronic diseases. The EPIC project began in 1992 and includes 520000 participants recruited from twenty-three administrative centres (reclassified into twenty-seven centres according to geographical region) in ten Western European countries: Greece, Spain, Italy, France, Germany, the Netherlands, the UK, Denmark, Sweden and Norway. Participants were mostly recruited from the general population, with some exceptions: women attending mammography screening (one centre in the Netherlands and one in Italy), women members of a health insurance for employees of the National Education System (France) and blood donors (some centres in Italy and Spain). Hence, nineteen of the twenty-seven EPIC regions recruited both women and men while eight regions recruited women only. In Oxford (UK), most participants were vegetarians or vegans and/or had a special interest in health, and are therefore evaluated separately (the 'UK Health-conscious' in contrast to the 'UK General population'). The EPIC project was approved by the ethical review boards of the International Agency for Research on Cancer (Lyon, France) and from all local centres. Written informed consent was obtained from all participants.

The calibration study was designed to account for random and systematic errors from food frequency-derived dietary data in EPIC and to enable examination of dietary data according to the same reference scale. The calibration study population was an age- and sex-stratified random sample of 36994 participants from the total EPIC cohort $(\sim 8 \%)$ and involved a single $24 \mathrm{~h}$ diet recall to be used as reference calibration method ${ }^{(20-22)}$. The results in the present paper are based on dietary data from the standardized $24 \mathrm{~h}$ diet recalls.

\section{Dietary assessment}

The $24 \mathrm{~h}$ diet recalls were collected using the standardized and computerized software EPIC-SOFT (now renamed
GloboDiet). The structure and functions of the software program have been described in detail elsewhere ${ }^{(20,23)}$. In short, EPIC-SOFT was administered by trained interviewers through face-to-face interviews in all countries except in Norway, where telephone interviews were performed. The interviews were structured into two steps: a first step where participants were asked to recall all foods and drinks consumed during the previous day; and a second step where they were asked to quantify and describe their intake. To standardize the memory aids used by the interviewers during the recall, eleven predefined food consumption occasions (FCO) were asked for, and information on all foods and drinks consumed were entered as one of the following FCO according to the participants' description: (i) before breakfast, (ii) breakfast, (iii) during morning, (iv) before lunch, (v) lunch, (vi) after lunch, (vii) during afternoon, (viii) before dinner, (ix) dinner, (x) after dinner and (xi) during evening. These FCO were defined to chronologically cover the different occasions of consumption during the day and consider the different food habits among the participating countries. Hence, a FCO could consist of single or combined food/s and/or drink/s. For each FCO, time of consumption was indicated per hour as integer values (e.g. 08.00, 09.00 hours, etc.) and each FCO, except for breakfast, lunch and dinner, could be selected several times during the day because of intakes in different hours (e.g. FCO 'during afternoon' consumed at both 14.00 and 17.00 hours). During the interview, participants were asked to list all foods and drinks consumed between waking up on the recall day and waking up on the next day, usually the interview day. However, interviews with regard to diet on Saturdays were conducted on Mondays in most countries for logistical reasons. The mean duration of the recalled day was always about $24 \mathrm{~h}^{(20)}$ and interviews were conducted over various seasons and days of the week. For calculation of energy intake, the EPIC Nutrient Database, developed to standardize the national nutrient databases across the ten EPIC countries, was used $^{(24,25)}$.

\section{Non-dietary variables}

The baseline examination in EPIC was performed during 1992-1998 and included detailed self-administered questionnaires on diet, medical history and lifestyle, which have been described elsewhere ${ }^{(19,26,27)}$. Through these questionnaires, data were collected on education level (none, primary, technical/professional, secondary, university), marital status (single, married/living together, divorced/separated and widowed), smoking (never, former, current), physical activity (inactive, moderately inactive, moderately active, active), diabetes (yes, no) and hyperlipidaemia (yes, no). To assess physical activity, the Cambridge physical activity index with four categories was used, which has been validated within the EPIC study and 
showed no significant heterogeneity by country ${ }^{(28)}$. For the calibration study, information on age, body weight and height were self-reported during the $24 \mathrm{~h}$ diet recall, and BMI was calculated as weight divided by the square of height. The mean time interval between the baseline examination and the $24 \mathrm{~h}$ diet recall varied between countries, from $1 \mathrm{~d}$ to 3 years ${ }^{(20)}$.

\section{Meal timetables and temporal distribution of food consumption occasions}

Meal timetables across the countries are displayed to illustrate time of consumption of meals (breakfast, lunch and dinner) and snacks (all other FCO) during the $24 \mathrm{~h}$ diet recall. Likewise, the proportion of FCO per hour for all EPIC countries are displayed in Fig. 1 to demonstrate temporal distribution. In line with our previous publication $^{(18)}$, we included all FCO in the analyses except for FCO consisting of water only (tap or mineral water), which were excluded. In addition, we also present Supplemental Fig. 1 (see online supplementary material) where only FCO containing $\geq 209 \cdot 2 \mathrm{~kJ}$ ( $\geq 50 \mathrm{kcal}$ ) are included to enable comparison with other studies using an energy content criterion to define an intake occasion ${ }^{(16)}$.

\section{Later:earlier energy intake ratio}

Timing of eating was examined as the later:earlier energy intake ratio, in line with previous research ${ }^{(12)}$. Earlier intakes were defined as 06.00-14.00 hours, and later intakes as $15.00-24.00$ hours. These time points were selected after studying the distribution of FCO and time of consumption of meals (Fig. 1 and Table 1) with the aim to produce two time periods that could be compared across a broad geographical span. This cut-off has previously been used to distinguish later $v$. earlier energy consumers ${ }^{(29)}$. Hence, a ratio of $<1.0$ indicates greater energy load earlier during the day (06.00-14.00 hours) and a ratio of $>1.0$ indicates greater energy load later during the day (15.0024.00 hours). To enable calculation of the ratio among all participants, earlier energy intakes that equalled $0 \mathrm{kcal}$ (i.e. the denominator) were replaced with $1 \mathrm{kcal}$.

\section{Statistical analysis}

Data are presented as mean, range, median, 1st-3rd quartile and mode. Type III tests of the generalized linear model procedure were used to evaluate associations between a priori selected variables (based on previous research and available data) and the later:earlier energy intake ratio, given all covariates in the model. The variables evaluated were country, age, educational level, marital status, smoking status, physical activity, BMI, prevalence of hyperlipidaemia and diabetes, day of recall and season. All variables were entered into the model as categorical variables except for age and BMI. The ratio was log-transformed before being entered into the model to correct for positive skewness. Adjusted $R^{2}$ is presented to display the variation in the ratio explained by the model, given all entered variables. Only data on participants who had complete covariate information were used in the model. All analyses are stratified by sex. Data were analysed using the statistical software package IBM SPSS Statistics version 21.0. Statistical significance was considered at $P<0.05$.

\section{Results}

\section{Study participants}

A total of 36020 participants (22985 women and 13035 men) with dietary data from the $24 \mathrm{~h}$ diet recall were included after exclusion of participants aged under 35 years or over 74 years due to low participation in these age groups ( $n$ 960) and of individuals with incomplete information ( $n$ 14). Mean (range) age for women and men ranged from $49 \cdot 0(35 \cdot 0-65 \cdot 5)$ and $50 \cdot 0(35 \cdot 2-65 \cdot 2)$ years (Bilthoven, the Netherlands) to $61 \cdot 4(45 \cdot 3-74 \cdot 2)$ and $64 \cdot 1$ (50.5-74.3) years (Malmö, Sweden), respectively. Mean BMI of women ranged from $22.9(14 \cdot 4-37 \cdot 6) \mathrm{kg} / \mathrm{m}^{2}$ (South of France, France) to $29.3(17.9-48.8) \mathrm{kg} / \mathrm{m}^{2}$ (Granada, Spain), and from $23.9(18.2-31.8) \mathrm{kg} / \mathrm{m}^{2}$ (UK Healthconscious) to $29 \cdot 3(20 \cdot 9-46 \cdot 2) \mathrm{kg} / \mathrm{m}^{2}$ (Granada, Spain) for men. For the Type III tests evaluating the association between the ratio and selected variables, 13132 women and 5680 men had complete covariate information.

\section{Meal timetables}

Overall, time of consumption of meals and snacks varied across the countries according to a south-north gradient (Table 1 and online supplementary material, Supplemental Table 1). As for breakfast, this was most often consumed at 07.00 hours in Sweden, Norway and France compared with 09.00 hours in Spain (Table 1). Following the time of breakfast, lunch was consumed earlier in the Nordic countries (12.00 hours) compared with Spain and Greece (14.00 hours). The greatest heterogeneity was observed for dinner, which was most frequently consumed between 16.00 and 19.00 hours in the Nordic countries compared with 20.00-21.00 hours in the Mediterranean countries. As for snacks, the largest difference was observed for the FCO 'during afternoon', where the most common time of consumption ranged from 14.00 hours in Norway to 18.00 hours in Spain and Greece, and for the FCO 'during evening', which ranged from 20.00 hours in Sweden to 24.00 hours in Spain.

\section{Temporal distribution of food consumption occasions}

In general, more distinct peak times for temporal distribution of FCO were observed in Mediterranean countries compared with Central/Northern European countries 

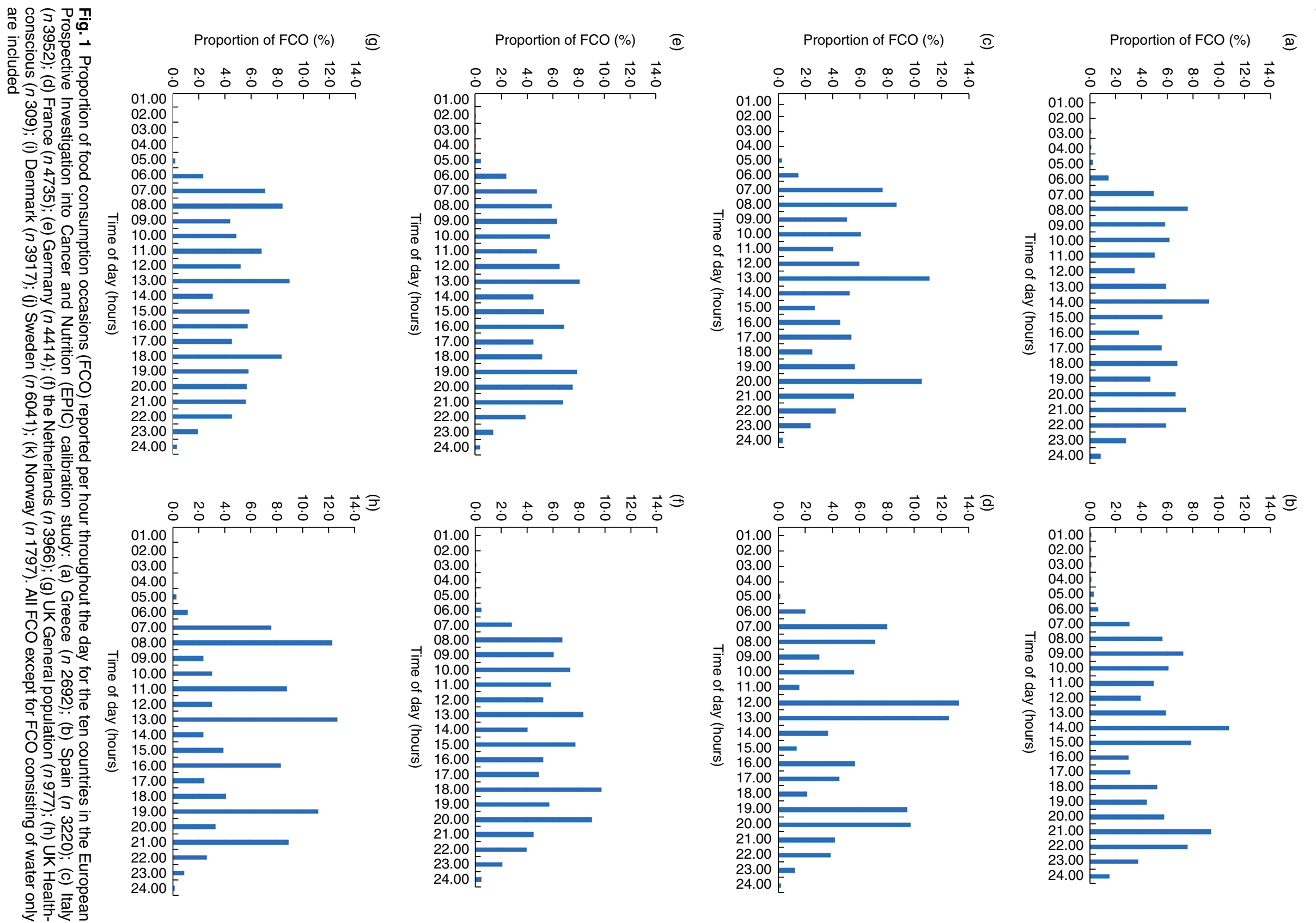

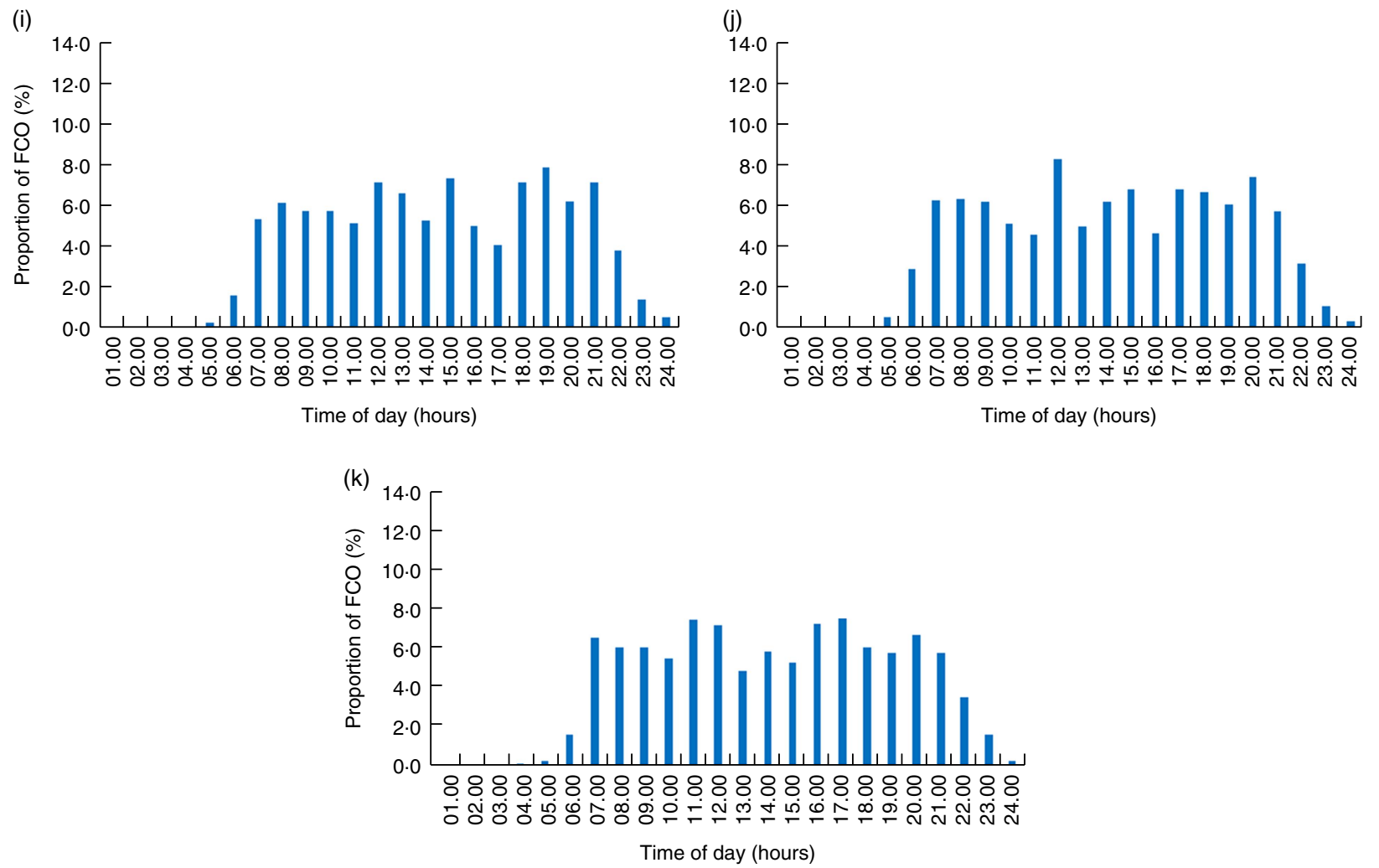

Fig. 1 (continued) Proportion of food consumption occasions (FCO) reported per hour throughout the day for the ten countries in the European Prospective Investigation into Cancer and Nutrition (EPIC) calibration study: (a) Greece ( $n$ 2692); (b) Spain ( $n$ 3220); (c) Italy ( $n$ 3952); (d) France ( $n$ 4735); (e) Germany ( $n$ 4414); (f) the Netherlands ( $n$ 3966); (g) UK General population ( $n$ 977); (h) UK Health-conscious ( $n$ 309); (i) Denmark ( $n$ 3917); (j) Sweden ( $n$ 6041); (k) Norway ( $n$ 1797). All FCO except for FCO consisting of water only are included

(Fig. 1). This was particularly prominent in France and Italy, where three peak times emerged at 07.00-08.00, 12.00-13.00 and 19.00-20.00 hours. In contrast, less pronounced peaks were observed in e.g. the Nordic countries, where FCO were more evenly spread across the day. Across all countries, the most defined peak time appeared at lunch time, with most countries displaying high frequency of FCO at 12.00-14.00 h. Similar patterns were observed when only FCO consisting of $\geq 209 \cdot 2 \mathrm{~kJ}$ ( $\geq 50 \mathrm{kcal}$ ) were included (see online supplementary material, Supplemental Fig. 1), although the peak times appeared more distinct after removal of small energy intakes. Temporal distribution of energy intake is presented in Supplemental Fig. 2.

\section{Later:earlier energy intake ratio}

A south-north gradient for the later:earlier energy intake ratio emerged, with Mediterranean countries (median ratio of 0.76 ) demonstrating a lower ratio compared with Central and Northern European countries (median ratio of 1.13 and $1 \cdot 15$, respectively). For women, the median ratio ranged from 0.68 in France to 1.39 in Norway (Table 2), and for men from 0.71 in Greece to 1.35 in the Netherlands
(Table 3). In addition, geographical differences for the ratio were observed within countries, with the ratio ranging from e.g. 0.66 (Navarra) to 2.04 (Granada) among women and from 0.72 (Navarra) to 2.58 (Granada) among men in Spain. In contrast, smaller within-country variations were observed for e.g. women in France (range 0.63-0.72) and men in Italy (range $0.73-0.88$; see online supplementary material, Supplemental Tables 2 and 3).

Among women, the following variables were associated with the ratio: country $(P<0 \cdot 001)$, age $(P<0 \cdot 001)$, education $(P<0.001)$, marital status $(P=0.021)$, smoking $(P<0.001)$, day of recall $(P<0.001)$ and season $(P=0.024$; Table 2). Importantly, female later energy consumers (i.e. ratio $>1.0$ ) tended to be from Central/Northern Europe, younger, have higher educational level, be current smokers, and to have captured a weekend day (i.e. SaturdaySunday) and the summer season during the recall interview. For men, statistically significant associations with the ratio were found for: country $(P<0.001)$, age $(P<0.001)$, education $(P<0 \cdot 001)$, smoking $(P<0 \cdot 001)$, physical activity $(P=0 \cdot 020)$, BMI $(P<0 \cdot 001)$ and day of recall $(P<0 \cdot 001$; Table 3$)$. Hence, importantly, male later energy consumers were more likely to be from Central/ Northern Europe, younger, to have higher educational 
৪.8:88:88:8

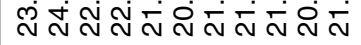

88888888888

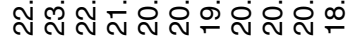

\section{8} ล

8888888888.

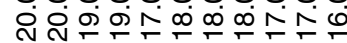

\section{৪৪৪৪৪৪৪৪৪৪৪} ஸ் 穴

\section{8}

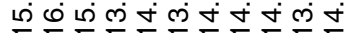

\section{৪৪৪৪৪৪৪৪৪৪০}

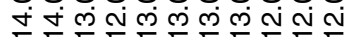

\section{৪৪৪৪৪৪৪৪৪৪৪}

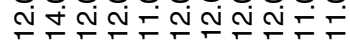

level, be current smokers, physically inactive and to have captured a weekend day during the recall interview. However, the models explained only 12.9 and $8.3 \%$ of the variation in the ratio among women and men, respectively.

\section{Discussion}

We set out to examine meal timetables and timing of eating across ten European countries. We found pronounced geographical differences across the countries, with later timing of meals and snacks but higher energy load earlier during the day in Mediterranean countries compared with Central/Northern European countries. Furthermore, among both women and men, we found that higher later compared with earlier energy load was associated with Central/Northern European countries, weekend days, and being younger, more educated and current smoker.

These results add to our previous report where we demonstrated that lunch provides 38-45\% of daily energy intake in Mediterranean countries and 16-27\% in Central/ Northern European countries ${ }^{(18)}$. In the current paper, we found that main meals are consumed later in most Mediterranean countries than in Central/Northern Europe (e.g. $09.00,14.00$ and 21.00 hours in Spain compared with 07.00, 12.00 and 16.00 hours in Norway, respectively). Still, within the Mediterranean countries, breakfast and lunch are consumed earlier in France than in Spain, Italy and Greece. This is in line with previous research demonstrating main meals to be consumed at 07.00, 12.00 and 20.00 hours in France ${ }^{(30)}$, compared with 09.00, 15.00 and 22.30 hours in Spain ${ }^{(31)}$. Furthermore, meal pattern analyses within the European Food Consumption Validation (EFCOVAL) study, conducted during 2007-2008, confirm our findings of more distinct peak times of eating in Mediterranean countries (represented by France in the EFCOVAL study) than in Central/Northern European countries (represented by the Netherlands, Norway, Belgium and the Czech Republic), where eating times were more spread throughout the day ${ }^{(30)}$. This finding is also in agreement with our previous report, where we found daily energy intake provided by snacks to be $10-20 \% v$. 23-35\% in the two regions, respectively, and the mean intake frequency to range from 4.9-5.0 (Greece and Italy) to $6 \cdot 8-7 \cdot 0$ (the Netherlands) FCO per day ${ }^{(18)}$, indicating more eating occasions between the main meals in Central/ Northern European than in Mediterranean countries.

Eating behaviour is a complex process influenced by social, cultural, biological and personal factors ${ }^{(32,33)}$. Historically, social constraints have determined the daily number of eating occasions and culture has dictated when eating is, or is not, appropriate ${ }^{(17)}$. In the present study, we found a near doubling of the later:earlier energy intake ratio from lowest to highest between the EPIC countries. 
Table 2 Later:earlier energy intake ratio across sociodemographic, lifestyle and health variables for women in the European Prospective Investigation into Cancer and Nutrition (EPIC) calibration study*

\begin{tabular}{|c|c|c|c|c|c|}
\hline & \multicolumn{4}{|c|}{ Later:earlier energy intake ratio } & \multirow[b]{2}{*}{$P$ value } \\
\hline & $n$ & $\%$ & Median & 1st-3rd quartile & \\
\hline Country & & & & & $<0.001$ \\
\hline Greece & 1368 & $6 \cdot 0$ & 0.73 & $0.40-1.76$ & \\
\hline Spain & 1443 & $6 \cdot 3$ & 0.91 & $0.53-2.90$ & \\
\hline Italy & 2510 & 10.9 & 0.83 & $0.57-1.20$ & \\
\hline France & 4735 & $20 \cdot 6$ & 0.68 & $0.48-0.99$ & \\
\hline Germany & 2147 & $9 \cdot 3$ & 0.93 & $0.61-1.46$ & \\
\hline The Netherlands & 2946 & $12 \cdot 8$ & 1.37 & $0.93-1.99$ & \\
\hline UK General population & 571 & 2.5 & 1.08 & $0.69-1.64$ & \\
\hline UK Health-conscious & 196 & 0.9 & 1.04 & $0.68-1.59$ & \\
\hline Denmark & 1994 & $8 \cdot 7$ & 1.28 & $0.87-1.95$ & \\
\hline Sweden & 3278 & 14.3 & 1.05 & $0.71-1.62$ & \\
\hline Norway & 1797 & $7 \cdot 8$ & 1.39 & $0.91-2.12$ & \\
\hline Age & & & & & $<0.001$ \\
\hline $35-44$ years & 2391 & $10 \cdot 4$ & $1 \cdot 13$ & $0.74-1.86$ & \\
\hline $45-54$ years & 8934 & 38.9 & 1.03 & $0.66-1.72$ & \\
\hline $55-64$ years & 8958 & 38.9 & 0.92 & $0.60-1.48$ & \\
\hline $65-74$ years & 2702 & 11.8 & 0.80 & $0.51-1.31$ & \\
\hline Educational level & & & & & $<0.001$ \\
\hline None/primary school & 7165 & 31.7 & 0.91 & $0.56-1.54$ & \\
\hline Secondary/technical school & 10534 & $46 \cdot 6$ & 1.02 & $0.66-1.63$ & \\
\hline University & 4905 & $21 \cdot 7$ & 0.95 & $0.61-1.55$ & \\
\hline Marital status & & & & & 0.021 \\
\hline Married or cohabitant & 15037 & $79 \cdot 0$ & 0.94 & $0.60-1.50$ & \\
\hline Single, divorced or widowed & 3988 & $21 \cdot 0$ & 0.95 & $0.60-1.54$ & \\
\hline Smoking status & & & & & $<0.001$ \\
\hline Never smoker & 13206 & $58 \cdot 4$ & 0.87 & $0.57-1.41$ & \\
\hline Former smoker & 5235 & $23 \cdot 2$ & 1.06 & $0.69-1.69$ & \\
\hline Current smoker & 4140 & $18 \cdot 3$ & $1 \cdot 20$ & $0.77-2.00$ & \\
\hline Physical activity & & & & & 0.559 \\
\hline Inactive & 2715 & $13 \cdot 8$ & 0.98 & $0.64-1.59$ & \\
\hline Moderately inactive & 7087 & $36 \cdot 1$ & 0.85 & $0.56-1.38$ & \\
\hline Moderately active & 8331 & $42 \cdot 5$ & 0.95 & $0.60-1.60$ & \\
\hline Active & 1480 & 7.5 & $1 \cdot 11$ & $0.70-1.73$ & \\
\hline BMI & & & & & 0.140 \\
\hline$<25.0 \mathrm{~kg} / \mathrm{m}^{2}$ & 12637 & $55 \cdot 0$ & 0.95 & $0.62-1.53$ & \\
\hline$\geq 25.0$ to $<30.0 \mathrm{~kg} / \mathrm{m}^{2}$ & 7135 & $31 \cdot 0$ & 1.01 & $0.62-1.67$ & \\
\hline$\geq 30.0 \mathrm{~kg} / \mathrm{m}^{2}$ & 3213 & $14 \cdot 0$ & 0.95 & $0.59-1.68$ & \\
\hline Hyperlipidaemia & & & & & 0.879 \\
\hline Yes & 2992 & $18 \cdot 2$ & 0.82 & $0.54-1.34$ & \\
\hline No & 13490 & $81 \cdot 8$ & 0.91 & $0.58-1.49$ & \\
\hline Diabetes & & & & & 0.881 \\
\hline Yes & 583 & $2 \cdot 6$ & 0.89 & $0.59-1.52$ & \\
\hline No & 21561 & 97.4 & 0.96 & $0.61-1.58$ & \\
\hline Day of recall & & & & & $<0.001$ \\
\hline Monday-Friday & 17124 & 74.5 & 0.95 & $0.62-1.53$ & \\
\hline Saturday-Sunday & 5861 & $25 \cdot 5$ & 1.01 & $0.59-1.79$ & \\
\hline Season & & & & & 0.024 \\
\hline Spring & 6810 & $29 \cdot 6$ & 0.92 & $0.59-1.53$ & \\
\hline Summer & 4723 & 20.5 & 1.02 & $0.66-1.66$ & \\
\hline Autumn & 5179 & 22.5 & 0.98 & $0.62-1.66$ & \\
\hline Winter & 6273 & $27 \cdot 3$ & 0.96 & $0.61-1.54$ & \\
\hline
\end{tabular}

*Type III tests of the generalized linear model procedure were used to evaluate associations between a priori selected variables and the ratio, given all covariates in the model. The ratio was log-transformed before entered into the model to correct for positive skewness. In total, the following number of women were missing covariate information: 381 women for educational status, 3960 women for marital status, 398 women for smoking status, 1808 women for physical activity, 6503 women for hyperlipidaemia status and 841 women for diabetes status. Only data on participants who had complete covariate information were used in the model (n 13132).

This suggests that cultural habits within a country are stronger predictors of temporal eating than any of our measured individual-level characteristics. Likewise, in the EFCOVAL study, country was found to be independently associated with all examined meal pattern aspects and to contribute the most to the variability in meal patterns ${ }^{(30)}$. Still, in the EPIC data, considerable variation in the ratio was observed across centres within the same country, e.g. 
Table 3 Later:earlier energy intake ratio across sociodemographic, lifestyle and health variables for men in the European Prospective Investigation into Cancer and Nutrition (EPIC) calibration study*

\begin{tabular}{|c|c|c|c|c|c|}
\hline & \multicolumn{4}{|c|}{ Later:earlier energy intake ratio } & \multirow[b]{2}{*}{$P$ value } \\
\hline & $n$ & $\%$ & Median & 1st-3rd quartile & \\
\hline Country & & & & & $<0.001$ \\
\hline Greece & 1324 & $10 \cdot 2$ & 0.71 & $0.43-1.92$ & \\
\hline Spain & 1777 & $13 \cdot 6$ & 0.88 & $0.54-2.65$ & \\
\hline Italy & 1442 & $11 \cdot 1$ & 0.85 & $0.61-1.24$ & \\
\hline France & - & - & - & - & \\
\hline Germany & 2267 & $17 \cdot 4$ & 1.00 & $0.67-1.54$ & \\
\hline The Netherlands & 1020 & $7 \cdot 8$ & 1.35 & $0.93-2.03$ & \\
\hline UK General population & 406 & $3 \cdot 1$ & 1.01 & $0.71-1.54$ & \\
\hline UK Health-conscious & 113 & 0.9 & 1.03 & $0.69-1.63$ & \\
\hline Denmark & 1923 & $14 \cdot 8$ & $1 \cdot 18$ & $0.81-1.80$ & \\
\hline Sweden & 2763 & $21 \cdot 2$ & 1.04 & $0.69-1.61$ & \\
\hline Norway & - & - & - & - & \\
\hline Age & & & & & $<0.001$ \\
\hline $35-44$ years & 1198 & $9 \cdot 2$ & $1 \cdot 18$ & $0.75-1.94$ & \\
\hline $45-54$ years & 4083 & 31.3 & $1 \cdot 12$ & $0.73-1.89$ & \\
\hline $55-64$ years & 5974 & 45.8 & 0.97 & $0.64-1.58$ & \\
\hline $65-74$ years & 1780 & 13.7 & 0.80 & $0.49-1.39$ & \\
\hline Educational level & & & & & $<0.001$ \\
\hline None/primary school & 5024 & 38.9 & 0.89 & $0.57-1.52$ & \\
\hline Secondary/technical school & 4645 & $36 \cdot 0$ & 1.08 & $0.71-1.74$ & \\
\hline University & 3233 & $25 \cdot 1$ & 1.09 & $0.71-1.85$ & \\
\hline Marital status & & & & & 0.098 \\
\hline Married or cohabitant & 7717 & 84.7 & 0.97 & $0.64-1.57$ & \\
\hline Single, divorced or widowed & 1389 & $15 \cdot 3$ & $1 \cdot 12$ & $0 \cdot 72-1 \cdot 82$ & \\
\hline Smoking status & & & & & $<0.001$ \\
\hline Never smoker & 4268 & $33 \cdot 1$ & 0.94 & $0.62-1.54$ & \\
\hline Former smoker & 5055 & $39 \cdot 2$ & 1.01 & $0.65-1.66$ & \\
\hline Current smoker & 3570 & $27 \cdot 7$ & $1 \cdot 10$ & $0.70-1.90$ & \\
\hline Physical activity & & & & & 0.020 \\
\hline Inactive & 2241 & $19 \cdot 2$ & $1 \cdot 14$ & $0.69-2.02$ & \\
\hline Moderately inactive & 3596 & 30.8 & 0.97 & $0.62-1.67$ & \\
\hline Moderately active & 4487 & 38.4 & 0.96 & $0.62-1.62$ & \\
\hline Active & 1357 & 11.6 & 1.03 & $0 \cdot 69-1.76$ & \\
\hline BMI & & & & & 0.002 \\
\hline$<25.0 \mathrm{~kg} / \mathrm{m}^{2}$ & 4140 & 31.8 & 1.00 & $0.68-1.59$ & \\
\hline$\geq 25.0$ to $<30.0 \mathrm{~kg} / \mathrm{m}^{2}$ & 6687 & $57 \cdot 2$ & 1.01 & $0.64-1.73$ & \\
\hline$\geq 30.0 \mathrm{~kg} / \mathrm{m}^{2}$ & 2208 & 18.9 & 1.00 & $0.61-1.80$ & \\
\hline Hyperlipidaemia & & & & & 0.542 \\
\hline Yes & 2774 & $30 \cdot 2$ & 0.98 & $0.63-1.65$ & \\
\hline No & 6401 & $69 \cdot 8$ & 0.97 & $0.62-1.71$ & \\
\hline Diabetes & & & & & 0.084 \\
\hline Yes & 664 & $5 \cdot 3$ & 0.89 & $0.57-1.41$ & \\
\hline No & 11979 & 94.7 & 1.01 & $0.65-1.69$ & \\
\hline Day of recall & & & & & $<0.001$ \\
\hline Monday-Friday & 9813 & $75 \cdot 3$ & 1.00 & $0.66-1.63$ & \\
\hline Saturday-Sunday & 3222 & $24 \cdot 7$ & 1.04 & $0 \cdot 62-1.89$ & \\
\hline Season & & & & & 0.586 \\
\hline Spring & 3553 & $27 \cdot 2$ & 0.98 & $0.63-1.65$ & \\
\hline Summer & 2785 & 21.4 & 1.05 & $0.69-1.78$ & \\
\hline Autumn & 2867 & $22 \cdot 0$ & 1.04 & $0.67-1.78$ & \\
\hline Winter & 3830 & 29.4 & 0.98 & $0.63-1.58$ & \\
\hline
\end{tabular}

*Type III tests of the generalized linear model procedure were used to evaluate associations between a priori selected variables and the ratio, given all covariates in the model. The ratio was log-transformed before entered into the model to correct for positive skewness. In total, the following number of men were missing covariate information: 133 men for educational status, 3929 men for marital status, 142 men for smoking status, 1354 men for physical activity, 3860 men for hyperlipidaemia status and 392 men for diabetes status. Only data on participants who had complete covariate information were used in the model ( $n 5680)$.

in Spain. This indicates both between- and within-country variation in timing of eating. Nevertheless, for the two UK centres, General population and Health-conscious, similar results were found for both timing of eating and the later: earlier energy intake ratio, although dinner was consumed somewhat later in the UK Health-conscious population. Furthermore, meal patterns have been reported to have seasonal and weekly variations, and to be influenced by 
age. For example, meals have been found to be shifted to the later part of the day on weekends compared with weekdays ${ }^{(34,35)}$, and the elderly have been reported to eat earlier in the day than younger individuals ${ }^{(36)}$. In addition, Leech et al. recently reported that individuals with a 'grazing' meal pattern, characterized by later and less distinct peak times of eating, were younger, had higher education (women) and were less likely to be married (men), compared with individuals with a 'conventional' or 'later lunch' pattern ${ }^{(37)}$. This is in agreement with our findings demonstrating a higher later:earlier energy intake ratio on weekends and among younger and higher educated individuals. Finally, and interestingly, we found that countries with later meal timetables (e.g. Mediterranean countries) had lower later:earlier energy intake ratio compared with countries with earlier meal timetables. This indicates that later timing of meals and snacks does not necessarily translate into greater energy load later during the day. However, the suggested misalignment between timing of eating and endogenous circadian systems ${ }^{(6,7)}$ may still apply, irrespective of temporal energy load. Future studies should evaluate how timing of eating, energy load across the day and irregularity in temporal distribution of eating affect metabolic circadian rhythm and diet-related disease risk.

We could not distinguish a clear and/or strong association between timing of eating and BMI, hyperlipidaemia or diabetes. Although eating behaviours are highly interrelated, consuming a greater proportion of daily energy intake at dinner and breakfast skipping have both been independently associated with obesity and the metabolic syndrome, also after adjusting for total energy intake ${ }^{(13)}$. For example, Aljuraiban et al. found that individuals who ate more frequently and consumed most of their energy intake earlier in the day (evening:morning energy intake ratio of $\leq 1.8$ ) had lower energy density and total energy intake, and higher nutrient quality, compared with participants with lower eating frequency and who consumed most of their energy intake later in the day (ratio of $>1 \cdot 8$ ). In addition, they found a positive association between BMI and evening:morning energy intake ratio also after adjusting for total energy intake ${ }^{(12)}$. Furthermore, in a 12 -week weight-loss trial among ninety women with metabolic syndrome, a high-energy breakfast was found to reduce weight, fasting glucose, insulin and TAG, and to increase satiety scores, more than a high-energy dinner under isoenergetic conditions ${ }^{(38)}$. Also, others have shown that later Spanish lunch eaters (after 15.00 hours), compared with earlier lunch eaters, lose less weight and have slower weight-loss trajectory ${ }^{(31)}$, and that eating a later lunch or snack is associated with impaired metabolism and decreased resting energy expenditure and diet-induced thermogenesis ${ }^{(39,40)}$. Thus, this suggests that a high energy intake earlier in the day may influence health more favourably than corresponding energy consumed later during the day. Even though high evening intake might reflect other lifestyle habits and/or food choices compared with high morning intake, a growing body of evidence indicates that circadian timing of eating affects body weight irrespective of total energy intake and may be a modifiable risk factor for diet-induced chronic disease.

The strengths of the present study include a large and diverse population sampled across several European countries concurrent with standardized and homogeneous dietary assessment methodology, which enabled an objective comparison of timing of eating across a broad geographical span. As for the limitations, the data herein were collected during 1995-2000 and are not nationally representative samples of the European general populations. However, the results may still demonstrate significant geographical differences between the countries due to the harmonized methodology used. In addition, our findings are confirmed by more recent analyses of meal patterns in adult European populations ${ }^{(30,31)}$. Second, the results are restricted to the methodology and definitions used through the earlier-later dichotomy approach, with earlier intakes defined as 06.00-14.00 hours. This cut-off was chosen as it encompasses the time of lunch for most countries and has been used previously to define earlier energy consumers ${ }^{(29)}$. Nevertheless, for some Mediterranean countries such as Spain, the cut-off was drawn during lunch hours, resulting in a higher ratio than if a later cut-off had been used. Still, the biological and metabolic implications of consuming a large proportion of daily energy intake later in the day should be the same irrespective of whether the energy load is labelled later lunch or earlier dinner. Third, participants missing complete covariate information were excluded. Fourth, the large sample size might have increased the number of statistically significant findings and the models explained only a small proportion of the variance in the ratio. Fifth, due to the use of single $24 \mathrm{~h}$ diet recalls and the cross-sectional design, we can only draw conclusions at the group level and interpret the results as hypothesis-generating that need to be evaluated in longitudinal and experimental settings. Finally, future research should examine what, and how, specific dietary factors relate to timing of eating, and could consider using data-driven approaches ${ }^{(37)}$ to examine patterns in timing of eating across meals and snacks.

\section{Conclusion}

We found pronounced differences in timing of eating across Europe, with later meal timetables and greater energy load earlier during the day in Mediterranean countries compared with Central and Northern European countries. More research is needed within the emerging field of chrono-nutrition to improve our understanding of the health implications of timing and patterning of eating throughout the day. 


\section{Supplementary material}

To view supplementary material for this article, please visit https://doi.org/10.1017/S1368980018002288

\section{Acknowledgements}

Financial support: This study was carried out with the financial support of the European Commission: Public Health and Consumer Protection Directorate 1993-2004; Research Directorate-General 2005, Ligue contre le Cancer (France); Société 3M (France); Mutuelle Générale de l'Education Nationale; Institut National de la Santé et de la Recherche Médicale (INSERM); Institute Gustave Roussy; German Cancer Aid; German Cancer Research Center; German Federal Ministry of Education and Research; Danish Cancer Society; Health Research Fund (FIS) of the Spanish Ministry of Health; Spanish Regional Governments of Andalucía, Asturias, Basque Country, Murcia (no. 6236) and Navarra and the Catalan Institute of Oncology; and ISCIII RETIC (RD06/0020), Spain; Cancer Research UK; Medical Research Council, UK; the Stroke Association, UK; British Heart Foundation; Department of Health, UK; Food Standards Agency, UK; the Wellcome Trust, UK; Greek Ministry of Health; Hellenic Health Foundation; Italian Association for Research on Cancer; Italian National Research Council, Regione Sicilia (Sicilian government); Associazione Italiana per la Ricerca sul Cancro-AIRC-Italy - ONLUS (Hyblean association for epidemiological research, NPO); Dutch Ministry of Health, Welfare and Sport; Dutch Prevention Funds; LK Research Funds; Dutch ZON (Zorg Onderzoek Nederland); World Cancer Research Fund (WCRF); Swedish Cancer Society; Swedish Research Council; Regional Government of Skane and the County Council of Vasterbotten, Sweden. The funders had no role in the design, analysis or writing of this article. Conflict of interest: None. Authorship: A.W. and H.B.F. initiated the study. E.H., A.W. and H.B.F. formulated the research questions, performed the analysis and wrote the manuscript taking into account comments from all co-authors. H.F., N.S, H.B., G.B., L.S. and E.W. contributed to the conception, analysis and interpretation of the data and drafting of the manuscript. All other co-authors were local EPIC collaborators involved in the collection of dietary data and other data. All authors read and approved the final version. Ethics of buman subject participation: The EPIC project was conducted according to the guidelines laid down in the Declaration of Helsinki and all procedures involving human subjects was approved by the ethical review boards of the International Agency for Research on Cancer (Lyon, France) and from all local centres. Written informed consent was obtained from all subjects.

\section{References}

1. World Health Organization (2014) Global Status Report on Noncommunicable Diseases 2014. Genera: WHO.

2. Benziger CP, Roth GA \& Moran AE (2016) The Global Burden of Disease Study and the preventable burden of NCD. Glob Heart 11, 393-397.

3. GBD 2015 Risk Factors Collaborators (2016) Global, regional, and national comparative risk assessment of 79 behavioural, environmental and occupational, and metabolic risks or clusters of risks, 1990-2015: a systematic analysis for the Global Burden of Disease Study 2015. Lancet 388, 1659-1724.

4. Garaulet M \& Gomez-Abellan P (2014) Timing of food intake and obesity: a novel association. Physiol Behav 134, $44-50$.

5. St-Onge MP, Ard J, Baskin ML et al. (2017) Meal timing and frequency: implications for cardiovascular disease prevention: a scientific statement from the American Heart Association. Circulation 135, e96-e121.

6. Marinac CR, Sears DD, Natarajan L et al. (2015) Frequency and circadian timing of eating may influence biomarkers of inflammation and insulin resistance associated with breast cancer risk. PLoS One 10, e0136240.

7. Morris CJ, Purvis TE, Mistretta J et al. (2017) Circadian misalignment increases C-reactive protein and blood pressure in chronic shift workers. J Biol Rhythms 32, 154-164.

8. Almoosawi S, Vingeliene S, Karagounis LG et al. (2016) Chrono-nutrition: a review of current evidence from observational studies on global trends in time-of-day of energy intake and its association with obesity. Proc Nutr Soc 75, 487-500.

9. Cahill LE, Chiuve SE, Mekary RA et al. (2013) Prospective study of breakfast eating and incident coronary heart disease in a cohort of male US health professionals. Circulation 128, 337-343.

10. Pan A, Schernhammer ES, Sun Q et al. (2011) Rotating night shift work and risk of type 2 diabetes: two prospective cohort studies in women. PLoS Med 8, e1001141.

11. Parkes KR (2002) Shift work and age as interactive predictors of body mass index among offshore workers. Scand $J$ Work Environ Health 28, 64-71.

12. Aljuraiban GS, Chan Q, Oude Griep LM et al. (2015) The impact of eating frequency and time of intake on nutrient quality and body mass index: the INTERMAP study, a population-based study. I Acad Nutr Diet 115, 528-536.e1.

13. Bo S, Musso G, Beccuti G et al. (2014) Consuming more of daily caloric intake at dinner predisposes to obesity. A 6year population-based prospective cohort study. PLoS One 9, e108467.

14. Seagle HM, Strain GW, Makris A et al. (2009) Position of the American Dietetic Association: weight management. $\mathrm{J} \mathrm{Am}$ Diet Assoc 109, 330-346.

15. Yumuk V, Tsigos C, Fried M et al. (2015) European guidelines for obesity management in adults. Obes Facts $\mathbf{8}$, 402-424.

16. Leech RM, Worsley A, Timperio A et al. (2015) Understanding meal patterns: definitions, methodology and impact on nutrient intake and diet quality. Nutr Res Rev 28, 1-21.

17. Bellisle F (2014) Meals and snacking, diet quality and energy balance. Physiol Behav 134, 38-43.

18. Huseinovic E, Winkvist A, Slimani N et al. (2016) Meal patterns across ten European countries - results from the European Prospective Investigation into Cancer and Nutrition (EPIC) calibration study. Public Health Nutr 19, 2769-2780.

19. Riboli E, Hunt KJ, Slimani N et al. (2002) European Prospective Investigation into Cancer and Nutrition (EPIC): study populations and data collection. Public Health Nutr 5, 1113-1124. 
20. Slimani N, Kaaks R, Ferrari P et al. (2002) European Prospective Investigation into Cancer and Nutrition (EPIC) calibration study: rationale, design and population characteristics. Public Health Nutr 5, 1125-1145.

21. Kaaks R, Plummer M, Riboli E et al. (1994) Adjustment for bias due to errors in exposure assessments in multicenter cohort studies on diet and cancer: a calibration approach. Am J Clin Nutr 59, 1 Suppl, 245S-250S.

22. Ferrari P, Day NE, Boshuizen HC et al. (2008) The evaluation of the diet/disease relation in the EPIC study: considerations for the calibration and the disease models. Int $\mathrm{J}$ Epidemiol 37, 368-378.

23. Slimani N, Deharveng G, Charrondiere RU et al. (1999) Structure of the standardized computerized 24-h diet recall interview used as reference method in the 22 centers participating in the EPIC project. European Prospective Investigation into Cancer and Nutrition. Comput Methods Programs Biomed 58, 251-366.

24. Deharveng G, Charrondiere UR, Slimani N et al. (1999) Comparison of nutrients in the food composition tables available in the nine European countries participating in EPIC. European Prospective Investigation into Cancer and Nutrition. Eur J Clin Nutr 53, 60-79.

25. Slimani N, Deharveng G, Unwin I et al. (2007) The EPIC nutrient database project (ENDB): a first attempt to standardize nutrient databases across the 10 European countries participating in the EPIC study. EurJ Clin Nutr 61, 1037-1056.

26. Haftenberger M, Schuit AJ, Tormo MJ et al. (2002) Physical activity of subjects aged 50-64 years involved in the European Prospective Investigation into Cancer and Nutrition (EPIC). Public Health Nutr 5, 1163-1176.

27. Friedenreich C, Cust A, Lahmann PH et al. (2007) Physical activity and risk of endometrial cancer: the European prospective investigation into cancer and nutrition. Int J Cancer 121, 347-355.

28. Peters T, Brage S, Westgate $\mathrm{K}$ et al. (2012) Validity of a short questionnaire to assess physical activity in 10 European countries. Eur J Epidemiol 27, 15-25.
29. Kaczmarek JL, Musaad SM \& Holscher HD (2017) Time of day and eating behaviors are associated with the composition and function of the human gastrointestinal microbiota. Am J Clin Nutr 106, 1220-1231.

30. Park MK, Freisling H, Huseinovic E et al. (2017) Comparison of meal patterns across five European countries using standardized 24-h recall (GloboDiet) data from the EFCOVAL project. Eur J Nutr 57, 1045-1057.

31. Garaulet M, Gomez-Abellan P, Alburquerque-Bejar JJ et al. (2013) Timing of food intake predicts weight loss effectiveness. Int J Obes (Lond) 37, 604-611.

32. De Castro JM (1997) Socio-cultural determinants of meal size and frequency. Br J Nutr 77, Suppl. 1, S39-S54.

33. Chiva M (1997) Cultural aspects of meals and meal frequency. Br J Nutr 77, Suppl. 1, S21-S28.

34. McCarthy S (2014) Weekly patterns, diet quality and energy balance. Physiol Behav 134, 55-59.

35. McHill AW, Phillips AJ, Czeisler CA et al. (2017) Later circadian timing of food intake is associated with increased body fat. Am J Clin Nutr 106, 1213-1219.

36. de Castro JM (2002) Age-related changes in the social, psychological, and temporal influences on food intake in free-living, healthy, adult humans. J Gerontol A Biol Sci Med Sci 57, M368-M377.

37. Leech RM, Worsley A, Timperio A et al. (2017) Temporal eating patterns: a latent class analysis approach. Int J Behav Nutr Phys Act 14, 3.

38. Jakubowicz D, Barnea M, Wainstein J et al. (2013) High caloric intake at breakfast vs. dinner differentially influences weight loss of overweight and obese women. Obesity (Silver Spring) 21, 2504-2512.

39. Bandin C, Scheer FA, Luque AJ et al. (2015) Meal timing affects glucose tolerance, substrate oxidation and circadianrelated variables: a randomized, crossover trial. Int $J$ Obes (Lond) 39, 828-833.

40. Romon M, Edme JL, Boulenguez C et al. (1993) Circadian variation of diet-induced thermogenesis. Am J Clin Nutr 57, 476-480. 